\title{
RIGHT-ORDERABLE DECK TRANSFORMATION GROUPS
}

\section{F. THOMAS FARRELL}

0. Introduction. Let $p: E \rightarrow B$ be a regular covering space such that $E$ is path connected, and $B$ is a Hausdorff, paracompact space with a countable fundamental group. Also let $\mathbf{R}$ denote the real line, and $q: B \times \mathbf{R} \rightarrow B$ be projection onto the first factor.

Question. Does there exist an embedding $f: E \rightarrow B \times \mathbf{R}$ such that the composite of $f$ with $q$ is $p$ ?

We show that the answer to this question is yes, if and only if $\pi_{1} B / p_{\#} \pi_{1} E$ is a right-orderable group.

In addition, if $B$ happens to be a manifold and $\pi_{1} B / p_{\#} \pi_{1} E$ is right orderable, then we show that $B \times \mathbf{R}$ can be foliated so that at least one of its leaves is a one-to-one continuous image of $E$, and the remaining leaves are one-to-one continuous images of intermediate covering spaces of $B$.

Rubin [10] had previously answered an important case of this Question. Namely he considered the universal cover of any space homotopically equivalent to a countable wedge of circles. Rubin's covering space result played a key role in the proof by R. D. Edwards and R. T. Miller [3] that cell-like closed-0-dimensional decompositions of $R^{3}$ are $R^{4}$ factors. Also Edwards and Miller extended Rubin's result to answer the above Question when $\pi_{1} B / p_{\#} \pi_{1} E$ is a countable free group.

\section{Preliminary facts about right-orderable groups.}

1.1. Definition. A right-ordered group is a pair $(G,>)$ where $G$ is a group, and $>$ is a total order on $G$, such that for all $x, y$, and $z$ in $G, x>y$ implies that $x z>y z$. A group $G$ is right-orderable, if there exists an order $>$ such that $(G,>)$ is a right-ordered group.

The following basic facts about right-orderable groups can be found in [1] and [4].

1.2. Right-orderable groups are torsion-free.

1.3. Any free group is right-orderable. Also any free abelian group is right-orderable.

Received by the editors on August 23, 1974, and in revised form on May 1, 1975. 
1.4. Any extension of a right-orderable group by a right-orderable group is right-orderable.

1.5. Example. By 1.3 and 1.4, the fundamental group of any closed 2-dimensional manifold, other than the sphere and the projective plane, is a right-orderable group.

We will need the next result in $\$ 2$.

1.6. LemMA. If $S$ is a countable, totally ordered set, then there is an order-preserving injection $f: S \rightarrow \mathbf{R}$, such that the image of $f$ is a discrete subspace of $\mathbf{R}$.

Proof. By adjoining two extra elements $\pm \infty$ to $S$, we can form a totally ordered set $S^{\prime}$, with maximal element $+\infty$ and minimal element $-\infty$, into which $S$ order-preservingly injects. Hence we may as well assume that $S$ has both a maximal and a minimal element.

Let $x_{0}, x_{1}, x_{2}, \cdots$ be an enumeration of the elements of $S$, such that $x_{0}$ is its minimal element, and $x_{1}$ is its maximal element; let $S_{n}=$ $\left\{x_{i} \mid i \leqq n\right\}$. For each integer $n \geqq 2$, let $x_{n}{ }^{-}$be the largest element in $S_{n}$ which is smaller than $x_{n}$, and let $x_{n}{ }^{+}$be the smallest element in $S_{n}$ which is larger than $x_{n}$.

Denote the Cantor middle-third set by $C$. Then $[0,1]-C$ is the disjoint union of a collection $I$ of open intervals. If $I$ is in $I$, we denote its length by $|I|$ and its midpoint by $b_{I}$. For each integer $n \geqq 2$, let

$$
A_{n}=\left\{I \in g|| I \mid=3^{1-n}\right\} \text { and } B_{n}=\left\{b_{I} \mid I \in A_{n}\right\} .
$$

In addition, define

$$
B_{0}=\{-2\}, B_{1}=\{2\} \text {, and } B=\bigcup_{n \geqq 0} B_{n} \text {. }
$$

Note that $B$ is a discrete subset of $\mathbf{R}$. Also notice that if $n>1$, and

$$
x, y \in \bigcup_{n>i} B_{i} \text { with } x>y,
$$

then there exists an element $z$ in $B_{n}$ such that $x>z>y$.

We now inductively define an order-preserving function $f: S \rightarrow B$ such that $f\left(x_{n}\right) \in B_{n}$. Start by putting $f\left(x_{0}\right)=-2$ and $f\left(x_{1}\right)=2$. If $f\left(x_{0}\right), f\left(x_{1}\right), \cdots, f\left(x_{n-1}\right)$ have already been defined, then let $f\left(x_{n}\right)$ be the smallest element $b$ in $B_{n}$ such that $f\left(x_{n}{ }^{+}\right)>b>f\left(x_{n}{ }^{-}\right)$.

The remainder of this section will be used only in $\S 3$.

1.7. Definition. An order $>$ on a set $S$ is said to be fine if, for each pair of points $x>y$, there exists a third point $z$ such that $x>z>y$, and $S$ contains neither a first nor a last point. 
1.8. LeMmA. If $\mathrm{S}$ is a countable set and $>$ is a fine total order on $S$, then there exists an order-preserving injection $f: S \rightarrow \mathbf{R}$ whose image is dense in $\mathbf{R}$.

The proof of this fact is left to the reader. It is similar to and easier than the proof of Lemma 1.6.

1.9. Lemma. If $(G,>)$ is a countable, right-ordered group, then there exists an order-preserving monomorphism $f: G \rightarrow H$ where $(H,>)$ is a countable right-ordered group and $>$ is a fine order.

Proof. Let $\mathbf{Q}$ be the additive group of rational numbers, then $H$ can be chosen to be the direct sum $G \oplus \mathbf{Q}$ lexicographically ordered, i.e., $(a, b)>(c, d)$ if and only if either $a>c$, or $a=c$ and $b>d$. And we can take $f(x)$ to be $(x, 0)$.

In the next lemma, $G$ has the discrete topology.

1.10. Lemma. If $G$ is a countable, right-orderable group, then $\mathbf{R}$ has a right G-space structure with at least one of its isotropy subgroups trivial.

Proof. Let $>$ be an order on $G$ so that $(G,>)$ is right-ordered. By Lemma 1.9, it suffices to consider the case when $>$ is fine. Also, by Lemma 1.8, we can identify $G$ with a dense subset of $R$. We proceed to define, for each $x$ in $G$, a homeomorphism $f(, x): \mathbf{R} \rightarrow \mathbf{R}$. For $r$ in $G$, define $f(r, x)$ to equal $r x$. Since $f(, x): G \rightarrow G$ is an orderpreserving bijection, and $G$ is dense in $R, f(, x)$ has a unique extension to a homeomorphism of $\mathbf{R}$. And it is easy to check that $f: \mathbf{R} \times$ $G \rightarrow \mathbf{R}$ is a $G$-space.

2. The main result. We begin by fixing some notation and assumptions to be used throughout this section. Let $E$ be a path connected space with base point $e_{0}$, and $p: E \rightarrow B$ a regular covering space; i.e., a principal bundle with discrete structure group. (See [11], page 70, for this definition.) Assume that $B$ is a Hausdorff paracompact space with a countable fundamental group and base point $b_{0}=p\left(e_{0}\right)$. Use $G$ to denote $\pi_{1}\left(B, b_{0}\right) / p_{\#} \pi_{1}\left(E, e_{0}\right)$. Then we identify $G$ with the group of deck transformations as follows. Let $T$ be a deck transformation and choose a path $\alpha$ from $e_{0}$ to $T\left(e_{0}\right)$. Then $p$ composed with $\alpha$ is some closed curve $\gamma$ in $B$ based at $b_{0}$. The map which sends $T$ to the equivalence class represented by $\gamma$ in $G$ is our posited isomorphism. Finally, if $x \in G$ and $S$ is either a subset or a point of $E$, then $x S$ denotes the image of $S$ under the action of $x$. 
2.1. Lemma. If there exists a continuous function $h: E \rightarrow \mathbf{R}$ such that the map $f: E \rightarrow B \times \mathbf{R}$ defined by $f(a)=(p(a), h(a))$ is an injection, then $G$ is right-orderable. If, in addition, the image of $f$ is a closed subset of $B \times \mathbf{R}$, then $G$ is either trivial or infinite cyclic.

Proof. Define a total order on $G$ as follows: $x>y$, if and only if $h\left(x e_{0}\right)>h\left(y e_{0}\right)$. We proceed, via proof by contradiction, to show that $(G,>)$ is a right-ordered group. Thus assume that $x, y$, and $z$ are elements in $G$ such that $x>y$, but $y z>x z$.

Let $\gamma$ be a loop in $B$ based at $b_{0}$ whose equivalence class in

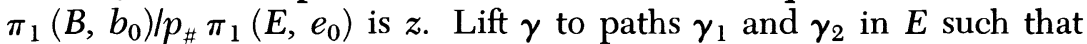
$\gamma_{1}(0)$ is $x e_{0}$ and $\gamma_{2}(0)$ is $y e_{0}$; then $\gamma_{1}(1)$ is $x z e_{0}$ and $\gamma_{2}(1)$ is $y z e_{0}$.

Consider the function $\ell:[0,1] \rightarrow R$ defined by $\ell(t)=h \gamma_{2}(t)-h \gamma_{1}(t)$. Since $\ell(1)>0>\ell(0)$, there exists a real number $t_{0}$ such that $\ell\left(t_{0}\right)$ is zero. Therefore $f \gamma_{1}\left(t_{0}\right)$ equals $f \gamma_{2}\left(t_{0}\right)$; hence, $\gamma_{1}\left(t_{0}\right)$ equals $\gamma_{2}\left(t_{0}\right)$. But two liftings of $\gamma$ which agree at one point must agree everywhere. This implies that $x$ equals $y$, which is the desired contradiction.

Now we continue under the added assumption that the image of $f$ is closed. Therefore $\varphi: G \rightarrow \mathbf{R}$ defined by $\varphi(x)=h\left(x e_{0}\right)$ is an orderpreserving bijection onto a closed subset $S$ of $\mathbf{R}$. Hence, either $>$ is fine, or the positive elements of $G$ form a well-ordered set. The second possibility can occur only when $G$ is either trivial or infinite cyclic. On the other hand, the first possibility implies that $S$ is perfect. And $S$ cannot be perfect since it is a countable set.

2.2 Leмма. If $B$ is a locally finite simplicial complex and $G$ is a right-orderable group, then there exists a continuous function $h: E$ $\rightarrow \mathbf{R}$ such that the map $f: E \rightarrow B \times \mathbf{R}$ defined by $f(a)=(p(a), h(a))$ is an embedding.

Proof. Put on $E$ the simplicial structure induced from the one of $B$ via $p$, i.e., the simplexes are the liftings to $E$ of the simplexes in $B$, and $p$ becomes a simplicial map. For each vertex $v$ of $B$, choose a point in $p^{-1}(v)$ and denote it by $v^{\prime}$.

Let $>$ be an order on $G$ such that $(G,>)$ is right-ordered. Then, by Lemma 1.6, there is an order-preserving injection $\varphi: G \rightarrow \mathbf{R}$ whose image is a discrete subset of $\mathbf{R}$.

We define $h$ on the vertices of $E$ as follows. For each vertex $v$ of $B$ and each element $x$ in $G$, let $h\left(x v^{\prime}\right)$ be $\varphi(x)$. Then we linearly extend $h$ to the rest of $E$. To be specific, consider the barycentric representation of a point $c$ in $E$, i.e.,

$$
c=t_{0} x_{0} v_{0}{ }^{\prime}+t_{1} x_{1} v_{1}{ }^{\prime}+\cdots+t_{n} x_{n} v_{n}{ }^{\prime},
$$


where $t_{i} \in[0,1], x_{i} \in G$, and the $v_{i}$ are vertices in $B$, such that $t_{0}+t_{1}+\cdots+t_{n}=1$ and $x_{0} v_{0}{ }^{\prime}, x_{1} v_{1}{ }^{\prime}, \cdots, x_{n} v_{n}{ }^{\prime}$ are the vertices of a simplex in $E$ containing $c$. Then define $h(c)$ to equal

$$
t_{0} \varphi\left(x_{0}\right)+t_{1} \varphi\left(x_{1}\right)+\cdots+t_{n} \varphi\left(x_{n}\right) .
$$

We will show that $f$ is an injection. (And leave the reader to check that $f$ is an embedding.) To do this, suppose that $c$ and $d$ are distinct elements in $E$ such that $p(c)=p(d)$. And let $\sigma$ be a simplex of $B$ containing $p(c)$. Then denote by $\sigma_{1}$ and $\sigma_{2}$ the simplexes in $E$ such that $c \in \sigma_{1}, d \in \sigma_{2}$, and $p\left(\sigma_{1}\right)=p\left(\sigma_{2}\right)=\sigma$. Thus there exists an element $x$ in $G$ such that $x \sigma_{1}=\sigma_{2}$ and $x^{-} \sigma_{2}=\sigma_{1}$. Let $e$ denote the identity element of $G$, then either $x>e$ or $x^{-1}>e$; hence, by symmetry, we may assume that $x>e$.

Let $v_{0}, v_{1}, \cdots, v_{n}$ be the vertices of $\sigma$, then there exists elements $x_{i}$ in $G$ such that the points $x_{i} v_{i}{ }^{\prime}$ are the verticies of $\sigma_{1}$; since $x \sigma_{1}=\sigma_{2}$, the vertices of $\sigma_{2}$ are the points $x x_{i} v_{i}{ }^{\prime}$ where $i=0,1 \cdots, n$. Thus $c$ can be written in barycentric co-ordinates as

$$
c=t_{0} x_{0} v_{0}{ }^{\prime}+t_{1} x_{1} v_{1}{ }^{\prime}+\cdots+t_{n} x_{n} v_{n}{ }^{\prime},
$$

where each $t_{i} \in[0,1]$ and $t_{0}+t_{1}+\cdots+t_{n}=1$; consequently,

$$
d=t_{0} x x_{0} v_{0}{ }^{\prime}+t_{1} x x_{1} v_{1}{ }^{\prime}+\cdots+t_{n} x x_{n} v_{n}{ }^{\prime},
$$

since $p(c)=p(d)$. Therefore,

$$
h(c)=t_{0} \varphi\left(x_{0}\right)+t_{1} \varphi\left(x_{1}\right)+\cdots+t_{n} \varphi\left(x_{n}\right)
$$

while

$$
h(d)=t_{0} \varphi\left(x x_{0}\right)+t_{1} \varphi\left(x x_{1}\right)+\cdots+t_{n} \varphi\left(x x_{n}\right) .
$$

But $x>e$ implies that $x x_{i}>x_{i}$, and $\varphi\left(x x_{i}\right)>\varphi\left(x_{i}\right)$ for $i=0, \cdots$, $n$; hence $h(d)>h(c)$. And this shows that $f$ is an injection.

2.3. THEOREM. There exists a continuous function $h: E \rightarrow \mathbf{R}$ such that the map $f: E \rightarrow B \times \mathbf{R}$ defined by $f(a)=(p(a), h(\vec{a}))$ is an embedding, if and only if $G$ is a right-orderable group.

Proof. There exists a connected, locally finite simplicial complex $X$ whose universal covering space $p^{\prime}: X^{\prime} \rightarrow X$ classifies principal $G$-bundles over Hausdorff, paracompact base spaces. (To see this, use [8, Th. 5.1] together with [2, Th. 7.5] and [9, Th. 1].) Thus there exist continuous functions $\ell: B \rightarrow X$ and $\ell^{\prime}: E \rightarrow X^{\prime}$, such that $p^{\prime} \ell^{\prime}=$ $\ell p$, and such that the function $k: E \rightarrow B \times X^{\prime}$ defined by $k(a)=$ $\left(p(a), \ell^{\prime}(a)\right)$ is an embedding onto a closed subset of $B \times X^{\prime}$. 
Assume that $G$ is right-orderable, then Lemma 2.2 is applicable to $p^{\prime}: X^{\prime} \rightarrow X$. Thus we obtain a continuous function $h^{\prime}: X^{\prime} \rightarrow \mathbf{R}$ such that the map $f^{\prime}: X^{\prime} \rightarrow X \times \mathbf{R}$ defined by $f^{\prime}(a)=\left(p^{\prime}(a), h^{\prime}(a)\right)$ is an embedding. Let $h$ be the composite of $\ell^{\prime}$ with $h^{\prime}$, then it is easily verified that $f$ is an embedding.

The other half of Theorem 2.3 is an immediate consequence of Lemma 2.1.

2.4. Corollary. There exists a continuous function $h: E \rightarrow \mathbf{R}$, such that the map $f: E \rightarrow B \times \mathbf{R}$ defined by $f(a)=(p(a), h(a))$ is a homeomorphism onto a closed subset of $B \times \mathbf{R}$, if and only if $G$ is either trivial or infinite cyclic.

Proof. If $G$ is trivial, then $h$ can be chosen to be identically zero. If $G$ is infinite cyclic, then the space $X$ used in the proof of Theorem 2.3 can be taken to be the circle $S^{1}$. In which case, $X^{\prime}$ is $R$, and we can choose $h$ to be $\ell^{\prime}$.

The other half of Corollary 2.4 is a consequence of Lemma 2.1.

3. A foliation of $\boldsymbol{M} \times \mathbf{R}$. We recall the definition of a codimension one foliation from [7]. (Lawson's paper is a good general reference on foliations.)

3.1. Definition. By a topological codimension one foliation of an $m$-dimension manifold $W$ we mean a decomposition of $W$ into a union of disjoint connected subsets $\left\{\mathcal{L}_{i}\right\}_{i \in I}$, called the leaves of the foliation, with the following property: Every point in $W$ has a neighborhood $U$ and a system of local coordinates $x=\left(x^{1}, \cdots, x^{m}\right): U \rightarrow R^{m}$ such that for each leaf $\mathcal{L}_{i}$, each component of $U \cap \mathcal{L}_{i}$ is described by an equation of the form $x^{m}=$ constant.

Let $p: E \rightarrow M$ be a regular covering space, where $M$ is a manifold, $E$ is connected, and $G=\pi_{1} M / p \# \pi_{1} E$ is right-orderable. Also let $q: M \times \mathbf{R} \rightarrow M$ denote projection onto the first factor.

3.2. THEOREM. There is a topological codimension one folidation of $M \times \mathbf{R}$ whose leaves $\mathcal{L}_{i}$ are indexed by some set I such that, to each $i \in I$, there corresponds an intermediate covering space $p_{i}: E_{i} \rightarrow M$, and a continuous bijection $f_{i}: E_{i} \rightarrow \mathcal{L}_{i}$ with $p_{i}=q f_{i}$; furthermore, there is at least one index $i$ with $p_{i}: E_{i} \rightarrow M$ equal to $p: E \rightarrow M$.

Proof. Put on $\mathbf{R}$ the right G-space structure posited in Lemma 1.10. (Since $M$ is a connected manifold, $G$ is countable; hence, Lemma 1.10 is applicable.) Then form the bundle $p^{\prime}: E^{\prime} \rightarrow M$ with fibre $R$ associated to the principal $G$-bundle $p: E \rightarrow M$. Note that $E^{\prime}$ is the quotient space of $\mathbf{R} \times E$ via the identifications $(r x, a)=(r, x a)$, where 
$r \in \mathbf{R}, x \in G$, and $a \in E$. (Here we have reversed, for convenience, the customary procedure in which the group of a principal bundle acts on the right side of its total space and on the left side of its associated fibre. See Chapter 4 of [6] for basic material on principal bundles.) It is easily seen that $E^{\prime}$ has a foliation possessing the properties described in Theorem 3.2. (The leaves of this foliation are in 1-1 correspondence with the orbits of the action of $G$ on $R$.)

Let Top $\mathbf{R}, \operatorname{Top}(0,1)$, and Top $[0,1]$ denote the order-preserving homeomorphisms of $\mathbf{R},(0,1)$, and $[0,1]$ respectively. Put on Top $[0,1]$ the topology of uniform convergence and topologize $\operatorname{Top}(0,1)$ by the natural identification of $\operatorname{Top}(0,1)$ with $\operatorname{Top}[0,1]$. Fix a homeomorphism $f: \mathbf{R} \rightarrow(0,1)$; identify Top $\mathbf{R}$ to $\operatorname{Top}(0,1)$ via conjugation with $f$; and thus induce a topology on Top $\mathbf{R}$. (This topology is independent of $f$.) Since $G$ acts on $\mathbf{R}$ via elements from Top $\mathbf{R}$, we can enlarge the structure group of $p^{\prime}: E^{\prime} \rightarrow M$ from $G$ to Top $\mathbf{R}$. But by Theorem 1.1.1 of [5] Top $R$ is contractible, hence $p^{\prime}: E^{\prime} \rightarrow M$ is topologically trivial; i.e., there exists a homeomorphism $k: E^{\prime} \rightarrow M \times \mathbf{R}$ with $p^{\prime}=q k$. Thus the foliation of $E^{\prime}$ induces, via $k$, the desired foliation of $M \times \mathbf{R}$.

\section{REFERENCES}

1. P. Conrad, Right-ordered groups, Michigan Math. J. 6(1959), 267-275.

2. A. Dold, Partitions of unity in the theory of fibrations, Annals of Math $\mathbf{7 8}$ (1963), 223-255.

3. R. D. Edwards and R. T. Miller, Cell-like closed-0-dimensional decompositions of $\mathbf{R}^{3}$ are $\mathbf{R}^{4}$ factors, ( to appear).

4. L. Fuchs, Teilweise geordnete algebraische Strukturen', Vandenhoeck + Ruprecht in Göttingen, 1966.

5. M-E. Hamstrom, Homotopy in homeomorphism spaces, Top and PL, Bull. of Amer. Math. Soc. 80(1974), 207-230.

6. D. Husemoller, Fibre Bundles, McGraw-Hill, New York, 1966.

7. H. B. Lawson, Foliations, Bull. of Amer. Math. Soc. 80(1974), 369-418.

8. J. Milnor, Construction of universal bundles, II, Annals of Math. 63(1956), 430-436.

9. J. Milnor, On spaces having the homotopy type of a CW-complex, Trans. of Amer. Math. Soc. 90(1959), 272-280.

10. L. R. Rubin, A general class of factors of $E^{4}$, Trans. of Amer. Math. Soc. 166(1972), 215-224.

11. N. Steenrod, The Topology of Fibre Bundles, Princeton University Press, Princeton, 1951.

Pennsylvania State University, University Park, Pennsylvania 16802 
